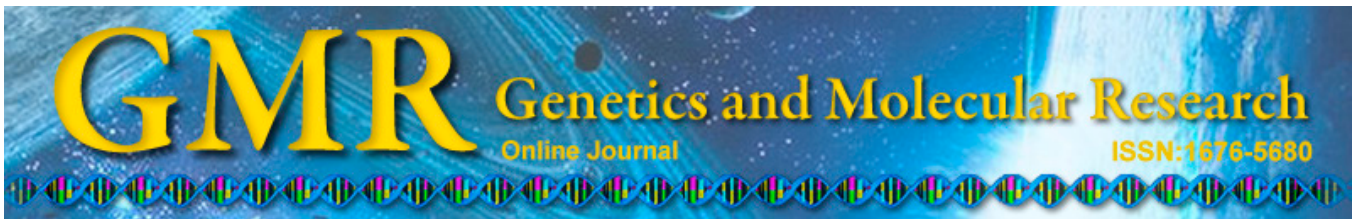

\title{
Isolation and characterization of cancer stem cells from medulloblastoma
}

\author{
J. Liu*, N. Chi*, J.Y. Zhang, W. Zhu, Y.S. Bian and H.G. Chen \\ Department of Neurosurgery, Yantai Yuhuangding Hospital, Yantai, China \\ *These authors contributed equally to this study. \\ Corresponding author: H.G. Chen \\ E-mail: ChenHongguang2014@163.com
}

Genet. Mol. Res. 14 (2): 3355-3361 (2015)

Received July 11, 2014

Accepted October 29, 2014

Published April 13, 2015

DOI http://dx.doi.org/10.4238/2015.April.13.15

\begin{abstract}
Brain cancer stem cells are a subset of tumor cells present in several types of brain tumor that evade treatment regimens and are responsible for tumor recurrence. Recent reports on several tumors have suggested that Hoechst 33342 dye exclusion is a powerful technique for isolating cancer stem cell-like side population (SP) cells. In the present study, we attempted to isolate the SP cells from medulloblastoma, a malignant brain tumor in children. The tumor samples obtained were subjected to fluorescence-activated cell sorting analysis for SP cell isolation. Further, the SP cells were characterized by a sphere-formation assay and analyzed for expression of stem cell genes by reverse transcriptionpolymerase chain reaction (RT-PCR). Using flow cytometry, we isolated $2.9 \%$ of cancer stem-like SP cells from malignant medulloblastoma, which was reduced to $0.4 \%$ in the presence of verapamil, an inhibitor of $\mathrm{ABC}$ transporter. These SP cells undergo rapid proliferation and have a high tendency to form tumor spheres when compared with non-SP cells. Further, RT-PCR analysis revealed that the isolated SP cells have increased expression of neural stem cell markers such as nestin, Notch1, and the $\mathrm{ABC}$ transporter protein $\mathrm{ABCG} 2$. Therefore, our findings suggest that SP cells of medulloblastoma share the characteristics of cancer stem cells.
\end{abstract}


The increased expression of stem cell markers and ABCG2 protein may function collectively and be responsible for drug and apoptosis resistance, as well as tumor recurrence and invasion.

Key words: Cancer stem cells; Medulloblastoma; Side population; Multidrug resistance; Chemotherapy

\section{INTRODUCTION}

The leading cause of cancer mortality in children is brain tumors, which mostly consist of morphologically diverse cells that express a wide range of neural lineage markers. Medulloblastoma (MB) is the most common malignant brain tumor in children (in contrast to adults) and the incidence mostly occurs between 4 and 7 years (Kaatsch et al., 2001; Louis et al., 2007; von Hoff and Rutkowski, 2012). The median life span of MB patients after diagnosis of the first symptoms ranges from 2 months to possibly years (Gerber et al., 2012). Despite advances in radio- or chemotherapy and surgery, the complete eradication of tumors is still complicated. It has been demonstrated in several solid tumors that treatment failure and tumor recurrence are due to the persistence of cancer stem cells (CSCs) (Fukaya et al., 2010).

The remarkable characteristic features of brain cancer stem cells (BCSCs) are their self-renewal, high potential for differentiation (into neurons, astrocytes, and oligodendrocytes), potency of tumor initiation, neurosphere formation, multidrug/apoptosis resistance, and expression of neural stem cell surface markers such as CD133 and nestin (Singh et al., 2004). The current conventional treatment strategies are able to target bulk tumor cells but do not affect the BCSCs, which ultimately results in treatment failure and tumor recurrence. Hence, the development of a new therapeutic strategy that effectively targets the BCSCs is an essential and urgent issue in the field of cancer treatment. Although several methods have been proposed for the isolation of BCSCs from brain tumors, Hoechst 33342 dye exclusion by flow cytometry has proven to be a powerful technique for isolating BCSCs, as well as other solid tumors.

The cancer cells that exclude Hoechst 33342 dye are called side population (SP) cells. These cells share all the characteristics features of BCSCs, such as self-renewal, reaction to tumor treatment (chemo- or radiotherapy), and stem cell gene expression to form various proteins (CD133, CD44, CD34, CD29, CD24, etc.). Further, these cells are highly multidrug- and apoptosis-resistant owing to overexpression of ATPase binding cassette (ABC) transporter proteins (such as $\mathrm{ABCB} 1, \mathrm{ABCC} 1, \mathrm{ABCG} 2$, and $\mathrm{BCRP} 1$ ) and Bcl-2, respectively.

SP cells have been identified and characterized in several solid tumors, including breast cancer, brain tumor, glioblastoma, gastrointestinal tumor, head and neck squamous cell carcinoma, hepatocellular cell lines, and primary cultures from neuroblastoma patients (Singh et al., 2003; Hirschmann-Jax et al., 2004). Therefore, characterization of SP cells in the tumor population will provide new insight into the mechanism of brain tumorigenesis, and help us to target the cells of tumor origin. Consequently, in the current study we attempted to isolate and characterize the SP cells from medulloblastoma, a malignant brain tumor in children.

\section{MATERIAL AND METHODS}

\section{Culture of tumor cells}

Tumor samples were obtained from patients $(\mathrm{N}=6$; age, 2.5-3 years; stage, malignant; 
infratentorial medulloblastoma) at the time of surgery. Tumors were washed, dissociated in oxygenated artificial cerebrospinal fluid, and subjected to enzymatic dissociation, as described previously (Singh et al., 2003). The tumor cells were then resuspended in Tris/sucrose $/ \mathrm{MgCl}_{2}$ (TSM) buffer containing serum-free neural stem cell medium, $20 \mathrm{ng} / \mathrm{mL}$ human recombinant epidermal growth factor, $20 \mathrm{ng} / \mathrm{mL}$ basic fibroblast growth factor, $10 \mathrm{ng} / \mathrm{mL}$ leukemia inhibitory factor, neuronal survival factor, and $60 \mathrm{~g} / \mathrm{mL} \mathrm{N}$-acetylcysteine. Red blood cells were removed using Lympholyte-M (Cedarlane, NC, USA).

\section{Study groups}

The medulloblastoma cell line MG-12 was divided into two groups: a control: group (MG-12 cells + Hoechst 33342 dye; $\mathrm{N}=6$, diplo/sample), and a drug-treated group (MG-12 cells + verapamil + Hoechst 33342 dye; $\mathrm{N}=6$, diplo/sample).

\section{Labeling with Hoechst 33342 dye}

Using a hemocytometer, 106 cells $/ \mathrm{mL}$ in 10\% Dulbecco's modified Eagle's medium were labeled with $5 \mu \mathrm{L} / \mathrm{mL}$ Hoechst 33342 stock (Sigma)-bis-benzimide, either with the dye alone or in combination with the drug $(0.8 \mu \mathrm{L} / \mathrm{mL}$ verapamil). After 90 -min incubation at $37^{\circ} \mathrm{C}$, cells were spun down $\left(2000 \mathrm{rpm}\right.$ for $10 \mathrm{~min}$ at $\left.4^{\circ} \mathrm{C}\right)$ and resuspended in $500 \mu \mathrm{L}$ Hanks' balanced salt solution containing $10 \mathrm{mM}$ 4-(2-hydroxyethyl)-1-piperazineethanesulfonic acid. Finally, cells were counterstained with $2 \mu \mathrm{g} / \mathrm{mL}$ propidium iodide (PI) per sample at $4^{\circ} \mathrm{C}$ to exclude the dead cells. Cells were filtered through a 50- $\mu$ m nylon mesh (BD Biosciences) to remove cell clumps into labeled fluorescence-activated cell sorting (FACS) tubes. The SP cells and main population (non-SP; NSP) cells were sorted using a flow cytometer (FACS Aria II; BD Biosciences). The Hoechst 33342 dye was excited at $355 \mathrm{~nm}$ and its dual wavelength fluorescence was analyzed (blue, $450 \mathrm{~nm}$; red, $675 \mathrm{~nm}$ ).

\section{Sphere-formation assay}

The sphere-formation assay was performed exactly as described previously (Fukaya et al., 2010). The SP and NSP cells sorted from medulloblastoma samples were seeded at a low density of 20 cells/L and the number of spheres generated was counted after 7 days of culture.

\section{In vitro proliferation activity assay}

An in vitro proliferation activity assay was performed as described previously ( $\mathrm{Fu}-$ kaya et al., 2010). The sorted SP and NSP cells were seeded on a 96-well plate at $2 \times 10^{\circ}$ cells/ well $(\mathrm{N}=4)$, and incubated in a $\mathrm{CO}_{2}$ incubator. Cell proliferation activity was measured every day for 7 days. The optical density was determined at $450 \mathrm{~nm}$ and the mean values were used to produce the cell growth graphs.

\section{Quantitative real-time polymerase chain reaction (PCR)}

Total RNA was isolated from FACS-sorted SP and NSP cells using an RNAqueousMicroKit (Ambion). cDNA was synthesized using a cDNA synthesis kit (Bioline). Real-time PCR was performed using 2-3 $\mu \mathrm{L}$ cDNA and $2 X$ TaqMan Gene Expression Mastermix in 50$\mu \mathrm{L}$ reaction volumes. Glyceraldehyde-3-phosphate dehydrogenase $(G A P D H)$ was used as the 
housekeeping gene. The primers were designed to encompass an exon junction to prevent templating from possibly contaminated genomic DNA. Primer sequences were: $A B C G 2$ : (F - AG C TGC AAG GAA AGA TCC AA and R - TCC AGA CAC ACC ACG GAT AA); for nestin: (F - TGG CTC AGA GGA AGA GTC TGA and R - TCC CCC ATT TAC ATG CTG TGA); for NOTCH1: (F - CAG GCA ATC CGA GGA CTA TG and R - CAG GCG TGT TGT TCT CAC AG); and for GAPDH: (F - ATG TCG TGG AGT CTA CTG GC and R - TGA CCT TGC CCA CAG CCT TG). The amplified products were separated by electrophoresis on ethidium bromidestained $1.2 \%$ agarose gels. Band intensity was measured using the Image $\mathrm{J}$ image analysis software (National Institutes of Health, Bethesda, MD, USA) from three independent experiments.

\section{Statistical analysis}

A one-way analysis of variance was performed to determine the significance between different treatment groups and individual Student $t$-tests were performed to compare the effect of different treatments between the SP and NSP populations. $* \mathrm{P}<0.05$ and $* * \mathrm{P}<0.01$ were considered to be statistically significant.

\section{RESULTS}

\section{Isolation of SP cells from a human medulloblastoma}

We analyzed the malignant human glioblastoma cell line for the presence of SP cells using Hoechst 33342 dye, which is a DNA-binding dye. Live cells were selected against PI, which was used to exclude the dead cells. Within the live cell population, we identified $2.9 \%$ of SP cells from medulloblastoma, as indicated by cells found towards the SP-violet region of the dot plot profile by FACS (Figure 1A; P2 gated region). The SP cell fraction was reduced to $0.4 \%$ (Figure 1B; P2 gated region) after treatment with verapamil, which blocks the ABC transporters (Goodell et al., 1996). Our findings suggest that the sorted SP cells are highly resistant to multidrug treatment, which may be due to the overexpression of $\mathrm{ABC}$ transporters, and hence treatment with verapamil significantly reduces the percentage of SP cells.
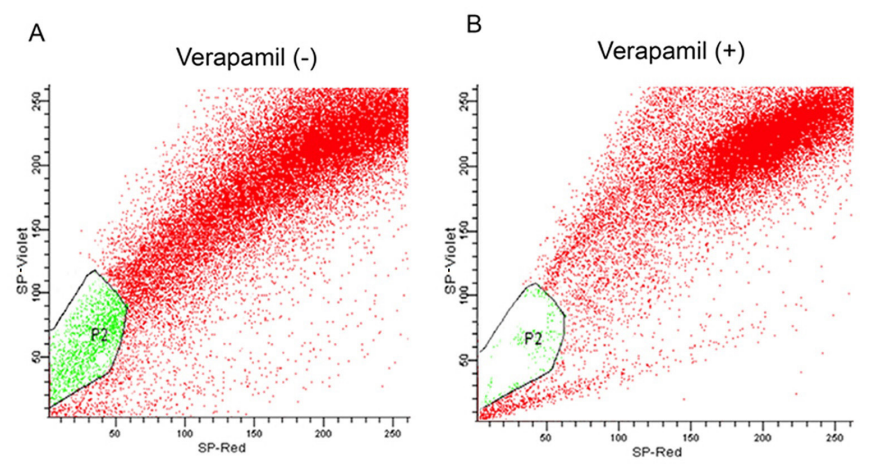

Figure 1. Analysis of side population (SP) cells in malignant brain tumor medulloblastoma. A. Cells were stained using Hoechst 33342 dye and analyzed by fluorescence-activated cell sorting (FACS). Medulloblastoma contained $2.9 \% \mathrm{SP}$ cells (P2 gated population) of the total cell population. B. The percentage of SP cells was significantly reduced to $0.4 \%$ (P2 gated region) in the presence of verapamil. 


\section{Sphere formation and high proliferation rate of SP cells}

The sorted SP cells and NSP cells were subsequently subjected to a sphere-formation assay. To compare their self-renewing capacities, both SP and NSP cells were cultured in neurosphere culture medium at low density $(20 \mathrm{cell} / \mathrm{s} / \mathrm{mL})$ and the number of spheres was counted after culture for 7 days. The rate and number of spheres generated by the SP cells were significantly higher than those generated by the NSP cells (Figure 2A). Subsequently, the in vitro cell proliferation assay revealed that SP cells of medulloblastoma increased cell proliferation from day 3 and they became confluent on day 7 when compared with NSP cells (Figure 2B).
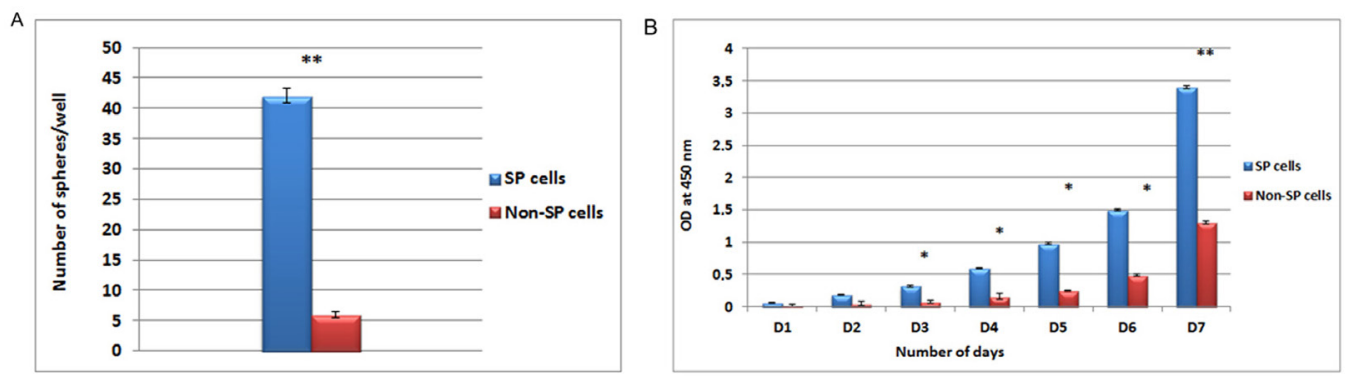

Figure 2. Sphere formation and increased cell proliferation properties of medulloblastoma side population (SP) cells. A. Quantification of number of spheres formed by sorted SP and non-SP (NSP) cells in neurosphere culture medium. After 7 days of culturing, the number of spheres formed by SP cells was significantly greater than by NSP cells. The bar represents standard deviation $* * \mathrm{P}<0.0001$. B. Cell proliferation rate of SP and NSP cells. The $\mathrm{x}$-axis represents time, while the $\mathrm{y}$-axis indicates the corresponding optical density $(\mathrm{OD})$ value at $450 \mathrm{~nm}(* \mathrm{P}<0.05 ; * * \mathrm{P}$ $<0.01)$. The bars represent standard deviation.

\section{Expression of stem cell gene analysis in MG-12 SP cells}

The sorted SP and NSP cells from medulloblastoma were analyzed for gene expression of $A B C G 2$ (ABC transporter), and stem cell genes nestin and NOTCH1, responsible for multidrug resistance and self-renewal capacity, respectively. Our RT-PCR analysis revealed that $A B C G 2$ and nestin gene expression were enhanced in SP cells more than in NSP cells (Figure 3A). In addition, NOTCH1 was highly expressed in SP cells, whereas the expression level was almost null in NSP cells (Figure 3A). The quantification graph clearly demonstrates that the expression levels of these genes were significantly higher in SP cells than in NSP cells (Figure 3B). GAPDH was used as a housekeeping gene.

\section{DISCUSSION}

Cancer stem cell theory highlights striking similarities of self-renewal, differentiation potential, and rapid proliferation mechanisms between stem cells and cancer stem cells (Reya et al., 2001). In addition, recent reports have suggested that BCSCs exhibit high tumorigenicity and multidrug and apoptosis resistance, and are therefore the major cause of treatment failure and tumor relapse. Therefore, identification and characterization BCSCs is urgently essential for understanding the molecular mechanisms behind brain tumorigenesis, and for 

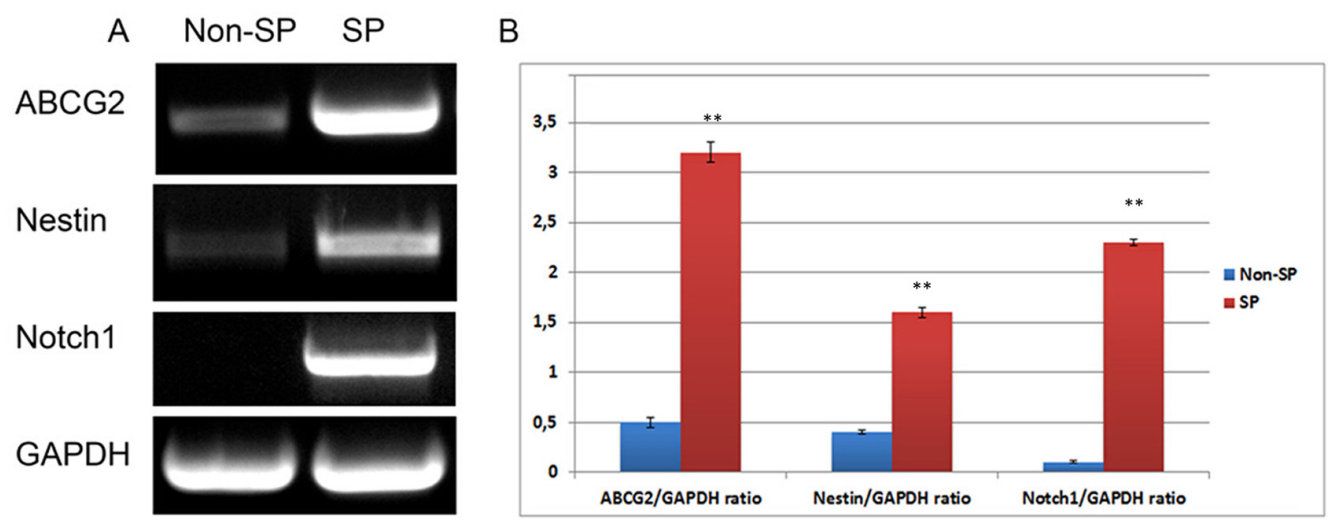

Figure 3. Expression of stem cell genes in side population (SP) and non-SP (NSP) cells. A. The elevated expressions of $A B C G 2, N E S T I N$, and NOTCH1 were detected by reverse transcription-polymerase chain reaction (RT-PCR). $G A P D H$ was used as the housekeeping gene. B. Quantification graph showing that the above-mentioned genes are significantly elevated in SP cells compared with NSP cells. The data presented in the graph are from two separate, independent experiments. Bars represent standard deviation (SD). ${ }^{* *}, \mathrm{P}<0.01$, compared with parental and NSP cells, respectively.

targeting and eradicating the key tumor cells that are responsible for treatment failure and tumor recurrence.

Cells that exclude Hoechst 33342 dye are called SP cells; they share many characteristics with cancer stem cells, such as tumor initiation and drug/apoptosis resistance, and express stem cell surface genes. SP cells were isolated from several solid tumors based on Hoechst 33342 dye efflux (Patrawala et al., 2005; Robey et al., 2008) due to overexpression of ABC transporters such as ABCB1 (MDR1), ABCC1, and ABCG2 (BCRP1), which are ultimately involved in multidrug resistance (Britton et al., 2012). In the present study, we isolated 2.9\% of SP cells from malignant brain tumor medulloblastoma of children using the Hoechst 33342 dye exclusion technique. The characteristic feature of Hoechst 33342 dye exclusion by SP cells involves the overexpression of the MDR1 transporter, specifically ABCG2, which is responsible for drug resistance in several solid tumors (Diestra et al., 2002; Lee et al., 2006). Treatment with verapamil reduced the SP cell population from 2.9 to $0.4 \%$. It has been reported that SP cells in most glioblastoma cell lines account for up to $1-2.5 \%$ of the cells and are significantly reduced following verapamil treatment (Patrawala et al., 2005; Fukaya et al., 2010). Further, we observed that SP cells are highly capable of self-renewal, as they rapidly generate more tumor spheres in neurosphere culture medium and have an increased capacity to proliferate in vitro. Similar to our findings, it has previously been shown that medulloblastoma cells are able to form more spheres when compared with astrocytoma and glioblastoma cells (Singh et al., 2003).

Moreover, several studies have demonstrated the overexpression of stem cell surface proteins such as nestin, Notch1, and CD133 in BCSCs (Singh et al., 2003; Calabrese et al., 2007; Bleau et al., 2009; Fukaya et al., 2010). In addition, the Notch and nestin pathways are known to promote the survival and proliferation of neural stem cells (Solecki et al., 2001). Our RT-PCR analysis showed greater expression of $A B C G 2$, nestin, and NOTCH1 genes in SP 
cells than in NSP cells. Hence, these results suggest that elevated expression of $A B C G 2$, nestin, and NOTCHI in SP cells acts as a crucial factor for drug resistance, massive proliferation of cancer cells, and tumor invasion.

Taken together, our findings clearly suggest that the failure of chemo- and drug therapy in cancer is mainly caused by the inability of SP cells to uptake the drugs owing to the overexpression of $\mathrm{ABC}$ transporters. Further, the elevated expression of ABCG2, nestin, and Notch1, which functionally interact with each other, promotes self-renewal, enhanced proliferation, and enhanced survival of SP cells. Therefore, further research into, and functional analysis of, the molecular mechanisms of BCSCs in tumorigenesis might produce novel therapeutic drugs that could target BCSCs and prevents tumor relapse.

\section{REFERENCES}

Bleau AM, Hambardzumyan D, Ozawa T, Fomchenko EI, et al. (2009). PTEN/PI3K/Akt pathway regulates the side population phenotype and ABCG2 activity in glioma tumor stem-like cells. Cell Stem. Cell 4: 226-235.

Britton KM, Eyre R, Harvey IJ, Stemke-Hale K, et al. (2012). Breast cancer, side population cells and ABCG2 expression. Cancer Lett. 323: 97-105.

Calabrese C, Poppleton H, Kocak M, Hogg TL, et al. (2007). A perivascular niche for brain tumor stem cells. Cancer Cell 11: 69-82.

Diestra JE, Scheffer GL, Catala I, Maliepaard M, et al. (2002). Frequent expression of the multi-drug resistance-associated protein $\mathrm{BCRP} / \mathrm{MXR} / \mathrm{ABCP} / \mathrm{ABCG} 2$ in human tumours detected by the BXP-21 monoclonal antibody in paraffinembedded material. J. Pathol. 198: 213-219.

Fukaya R, Ohta S, Yamaguchi M, Fujii H, et al. (2010). Isolation of cancer stem-like cells from a side population of a human glioblastoma cell line, SK-MG-1. Cancer Lett. 291: 150-157.

Gerber NU, von HK, von Bueren AO, Treulieb W, et al. (2012). A long duration of the prediagnostic symptomatic interval is not associated with an unfavourable prognosis in childhood medulloblastoma. Eur. J. Cancer 48: 2028-2036.

Goodell MA, Brose K, Paradis G, Conner AS, et al. (1996). Isolation and functional properties of murine hematopoietic stem cells that are replicating in vivo. J. Exp. Med. 183: 1797-1806.

Hirschmann-Jax C, Foster AE, Wulf GG, Nuchtern JG, et al. (2004). A distinct "side population" of cells with high drug efflux capacity in human tumor cells. Proc. Natl. Acad. Sci. U. S. A. 101: 14228-14233.

Kaatsch P, Rickert CH, Kuhl J, Schuz J, et al. (2001). Population-based epidemiologic data on brain tumors in German children. Cancer 92: 3155-3164.

Lee J, Kotliarova S, Kotliarov Y, Li A, et al. (2006). Tumor stem cells derived from glioblastomas cultured in bFGF and EGF more closely mirror the phenotype and genotype of primary tumors than do serum-cultured cell lines. Cancer Cell 9: 391-403.

Louis DN, Ohgaki H, Wiestler OD, Cavenee WK, et al. (2007). The 2007 WHO classification of tumours of the central nervous system. Acta Neuropathol. 114: 97-109.

Patrawala L, Calhoun T, Schneider-Broussard R, Zhou J, et al. (2005). Side population is enriched in tumorigenic, stemlike cancer cells, whereas ABCG2+ and ABCG2- cancer cells are similarly tumorigenic. Cancer Res. 65: 6207-6219.

Reya T, Morrison SJ, Clarke MF and Weissman IL (2001). Stem cells, cancer, and cancer stem cells. Nature 414: 105-111.

Robey RW, Shukla S, Finley EM, Oldham RK, et al. (2008). Inhibition of P-glycoprotein (ABCB1)- and multidrug resistance-associated protein 1 (ABCC1)-mediated transport by the orally administered inhibitor, CBT-1(R). Biochem. Pharmacol. 75: 1302-1312.

Singh SK, Clarke ID, Terasaki M, Bonn VE, et al. (2003). Identification of a cancer stem cell in human brain tumors. Cancer Res. 63: 5821-5828.

Singh SK, Hawkins C, Clarke ID, Squire JA, et al. (2004). Identification of human brain tumour initiating cells. Nature 432: 396-401.

Solecki DJ, Liu XL, Tomoda T, Fang Y, et al. (2001). Activated Notch2 signaling inhibits differentiation of cerebellar granule neuron precursors by maintaining proliferation. Neuron 31: 557-568.

von Hoff K and Rutkowski S (2012). Medulloblastoma. Curr. Treat. Options. Neurol. 14: 416-426.

Genetics and Molecular Research 14 (2): 3355-3361 (2015) 